Acta Crystallographica Section B

Structural

Science

ISSN 0108-7681

Editor: Carolyn P. Brock

\title{
The crystal structure of phenyl benzoate
}

\author{
J. M. Adams and S. E. Morsi
}

This electronic document was scanned from an archival copy of material deposited to accompany a paper published in an IUCr journal. In many cases the only accessible copy was a microfilm of a poor-quality original. 


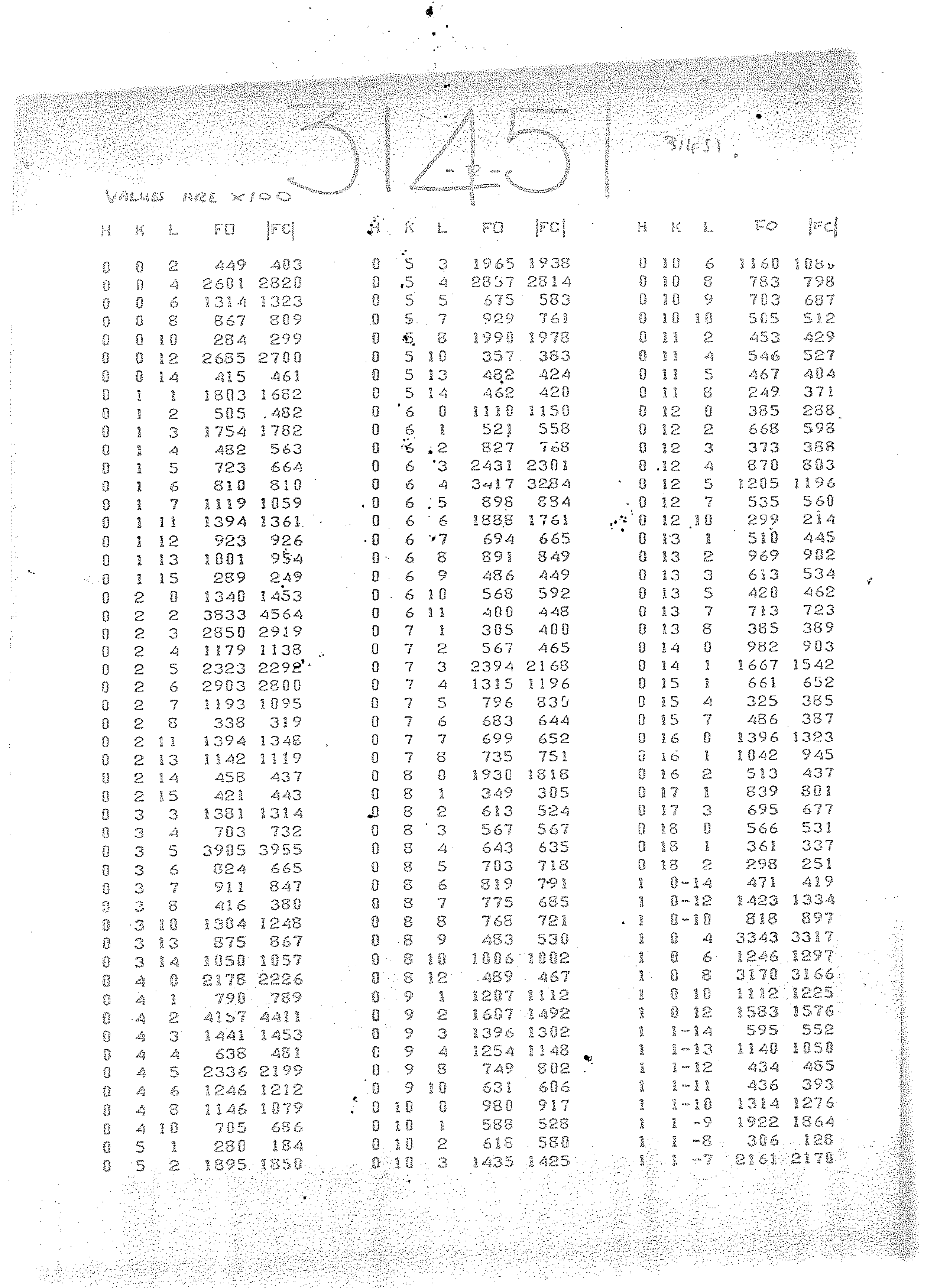




\begin{tabular}{|c|c|c|c|c|c|c|c|c|c|c|c|c|c|}
\hline$k$ & 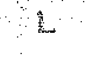 & 50 & $\mid \mathrm{F}$ & $H$ & K & $n$ & $\mid m C$ & & $\mathrm{H}$ & $K$ & $i$ & $\mathrm{E}=0$ & w \\
\hline$\hat{i}$ & -6 & 268 & 283 & 是 & 6 & 3084 & 355 & & $\hat{i}$ &  & -5 & 748 & 1723 \\
\hline$\hat{8}$ & $=-5$ & 276 & $25 \%$ & 1 & 7 & 363 & 285 & & $\stackrel{?}{\xi}$ & 6 & -4 & 1222 & 1249 \\
\hline t & -4 & 2059 & 2056 & $i$ & 8 & 579 & 536 & & 3 & 6 & -3 & 1273 & 1245 \\
\hline 1 & -3 & 553 & 192 & 3 & 9 & 35. & 427 & & $\hat{\mathbb{I}}$ & 6 & -2 & 35 & 283 \\
\hline I & $-?$ & 250 & 2791 & - & 310 & 3842 & 1799 & & $\mathfrak{a}$ & 6 & $-\frac{\bar{s}}{2}$ & 5374 & 1352 \\
\hline 1 & 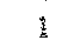 & 94 & 63 & 1 & $4-30$ & 903 & 884 & & 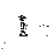 & 5 & $n$ & 232 & 233 \\
\hline 2 & 2 & 2195 & 2193 & 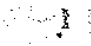 & $4:-9$ & 1954 & 3107 & & $\frac{1}{3}$ & 6 & 3 & 1606 & 989 \\
\hline$\stackrel{?}{q}$ & 3 & 3580 & 3550 & 3 & $4-8$ & 562 & $\$ 607$ & & 1 & 6 & 2 & 3479 & 3482 \\
\hline 1 & $\dot{A}$ & 1260 & $124 ?$ & 1 & $A-4$ & 3454 & 3485 &. & $\underline{2}$ & 6 & 3 & 3269 & 3277 \\
\hline s. & 5 & I4G2 & 1490 & 1 & $4-3$ & 599 & 2044 & * & 1 & 6 & 4 & 2596 & 2653 \\
\hline$\frac{3}{2}$ & 6 & 204 & 93 & $\frac{3}{2}$ & $\Rightarrow-2$ & 3869 & 3772 & & $\S$ & 6 & 5 & 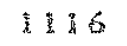 & 3242 \\
\hline$\sqrt[3]{1}$ & 7 & 5333 & 1282 & $\frac{n}{n}$ & $4^{*}-1$ & 909 & 866 & & $\tilde{s}$ & 6 & 6 & 599 & 5.44 \\
\hline$\hat{\mathfrak{z}}$ & 9 & 393 & 407 &  & 4 & $14 \Delta 1$ & 454 & & $\frac{2}{2}$ & 6 & 7 & 306 & 356 \\
\hline$i$ & 10 & 1250 & 1290 & $4^{2}$ & 2 & 877 & $7 \% 6$ & & 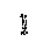 & 6 & 8 & 1260 & 1273 \\
\hline ? & $\frac{1}{3}$ & 1256 & 1168 & 3 & 4 & 1002 & 916 & & 1 & $7-$ & -10 & 495 & $45 \frac{1}{3}$ \\
\hline$\frac{\xi}{2}$ & 13 & 380 & 37.7 & 1. & 4 & 2355 & 2226 & & 2 & 7 & -9 & 807 & 823 \\
\hline & -12 & 895 & 92.6 & $\frac{3}{2}$ & 4 & 572 & $5: 3$ & & $\frac{3}{3}$ & 7 & -5 & 465 & 442 \\
\hline $2-$ & -10 & 5039 & 1038 & 1 & 4 & 889 & 817 & & $\tilde{i}$ & 7 & -4 & 1272 & 1254 \\
\hline 2 & -8 & 705 & $7: 5$ & $i$ & $B$ & 2540 & 2452 & & 1 & 7 & -3 & 1161 & 1137 \\
\hline 2 & -7 & 2238 & 2169 & 1 & 4 & 378 & 405 & & 1 & 7 & -2 & 1933 & 1874 \\
\hline 2 & -6 & 91.6 & 928 & i: & $\because 410$ & $9 \div 4$ & 981 & & 1 & 7 & -1 & 1705 & 1714 \\
\hline 2 & -5 & 2969 & 2929 & 1 & $\dot{4} 12$ & 983 & 1008 & & 1 & 7 & 0 & 234 & 210 \\
\hline 2 & -4 & 3550 & 1473 & 1 & 413 & 457 & 457 & & 1 & 7 & 1 & 1765 & 1357 \\
\hline 2 & -3 & 5037 & $512 \dot{7}$ & 1 . & 414 & 326 & 341 & & 1 & 7 & 2 & 2998 & 2969 \\
\hline e & 1 & 3764 & 3870 & 1 & $5-11$ & 363 & $\therefore \quad 434$ & & 1 & 7 & 3 & 256 & 169 \\
\hline 2 & 3 & 2646 & 2686 & 1 & $5-9$ & 394 & 334 & $\therefore$ & 1 & 7 & 4 & 1998 & 1144 \\
\hline 2 & 4 & 238 & 191. & 1 & $5-8$ & 742 & 774 & & $\frac{1}{2}$ & 7 & 9 & 516 & 542 \\
\hline 2 & 5 & 2386 & 2465 & $i$ & $5-5$ & 4802 & $4766^{\circ}$ & & 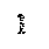 & 7 & 10 & 698 & 788 \\
\hline 2 & 6 & 1296 & 1242 & 3 & $5 .-4$ & 2117 & 2156 & &  & 7 & 23 & 334 & 240 \\
\hline 2 & 8 & 1228 & 1204 & $\$$ & $51-3$ & 2433 & 1462 & & $\overrightarrow{3}$ & B & -5 & 859 & 887 \\
\hline 2 & 9 & 596 & 656 & 1 & $5-2$ & 589 & 519 & & \pm & 8 & -9 & 433 & 458 \\
\hline 2 & 30 & 359 & 1189 & 1 & $5-1$ & 1851 & 1931 & & $\xi$ & 8 & -7 & 626 & 609 \\
\hline 2 & 13 & 349 & 378 & $\frac{1}{3}$ & 5 & 897 & 949 & & $\frac{1}{2}$ & 8 & -6 & 395 & 393 \\
\hline 2 & $\$ 2$ & 956 & 973 & $\frac{1}{2}$ & $5: 3$ & 3942 & 3935 & & 1 & 8 & -4 & 2096 & 5973 \\
\hline 2 & $3<$ & 291 & 282 & $\hat{2}$ & 5 & 2703 & 1766 & & 3 & 8 & -3 & 325 & 989 \\
\hline $3-$ & 喜㶻 & 932 & 954 & $\hat{\mathbf{1}}$ & 5 & 432 & 402 & & $\sqrt[3]{2}$ & 8 & -2 & 1925 & $\therefore 757$ \\
\hline $3-$ & 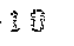 & 논 & 1. 165 & 1 & 5 & U. 32 & 455 & & 2 & 8 & $a n$ & 798 & 715 \\
\hline 3 & -9 & $3 \pm 56$ & 3225 & $\frac{3}{4}$ & 5 & 438 & 454 & & $\mathfrak{s}$ & 8 & 0 & 455 & $8 \mathrm{CO}^{2}$ \\
\hline 3 & -3 & 3665 & 257 & 重 & 5 & 373 & 592 & & 1 & 3 & : & 1320 & 1293 \\
\hline 3 & -5 & 2656 & 265 & 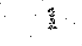 & 5 & $5 A G$ & 516 & $\therefore$ & 3 & $\mathrm{~B}$ & 2 & 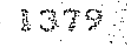 & 128 \\
\hline 3 & -3 & 2n: & 1926 & 3 & 5 & 725 & 755 & $\therefore$ & 紊 & $g$ & 3 & 1556 & $\triangle B A$ \\
\hline 3 & 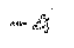 & 2279 & 2265 & 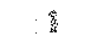 & 52 & 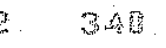 & 3,2 & & 2 &  & 5 & 57 & 561 \\
\hline 3 & -3 & $5 x 2$ & 5426 &  & 53 & 315 & 292 & & $\mathrm{~s}$ & 3 & 8 & 792 & 338 \\
\hline 3 & $-\frac{3}{z^{2}}$ & 1953 & 跑3 & $\frac{1}{5}$ & 524 & 396 & 456 & & $\$$ & $g$ & 20 & 545 & 532 \\
\hline 3 & $\hat{0}$ & 2530 & 3598 & 言 & $6-\frac{12}{12}$ & 574 & 582 & & $\underline{2}$ & & 10 & 423 & 429 \\
\hline 3 & $\hat{B}$ & 2375 & 2412 & i & $6-35$ & $A A B$ & 429 & & $\hat{3}$ & 9 & $-a_{2}$ & 1625 & 552 \\
\hline 3 & 2 & $\$ 539$ & 4736 & $\frac{3}{2}$ & $6-10$ & 3000 & 4021 & & $\stackrel{3}{11}$ & 9 & -3 & 330 & 252 \\
\hline 3 & 3 & 3727 & 3757 & s & $6 \cdot-8$ & 1690 & 3649 & 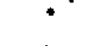 & $\$$ & 9 & -2 & 2432 & 2497 \\
\hline 3 & 4 & 893 & 90 &  & $6-7$ & 893 & 705 & $\because$ & 暠 & 9 & I & $m \mathrm{~g}$ & 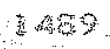 \\
\hline 3 & 5 & $54 B$ & 580 & $\sqrt[3]{2}$ & 60 & S4 & 825 & $\cdots{ }^{\circ}$ & $\therefore$ & 9 & 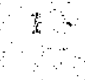 & 烈 & 373 \\
\hline
\end{tabular}




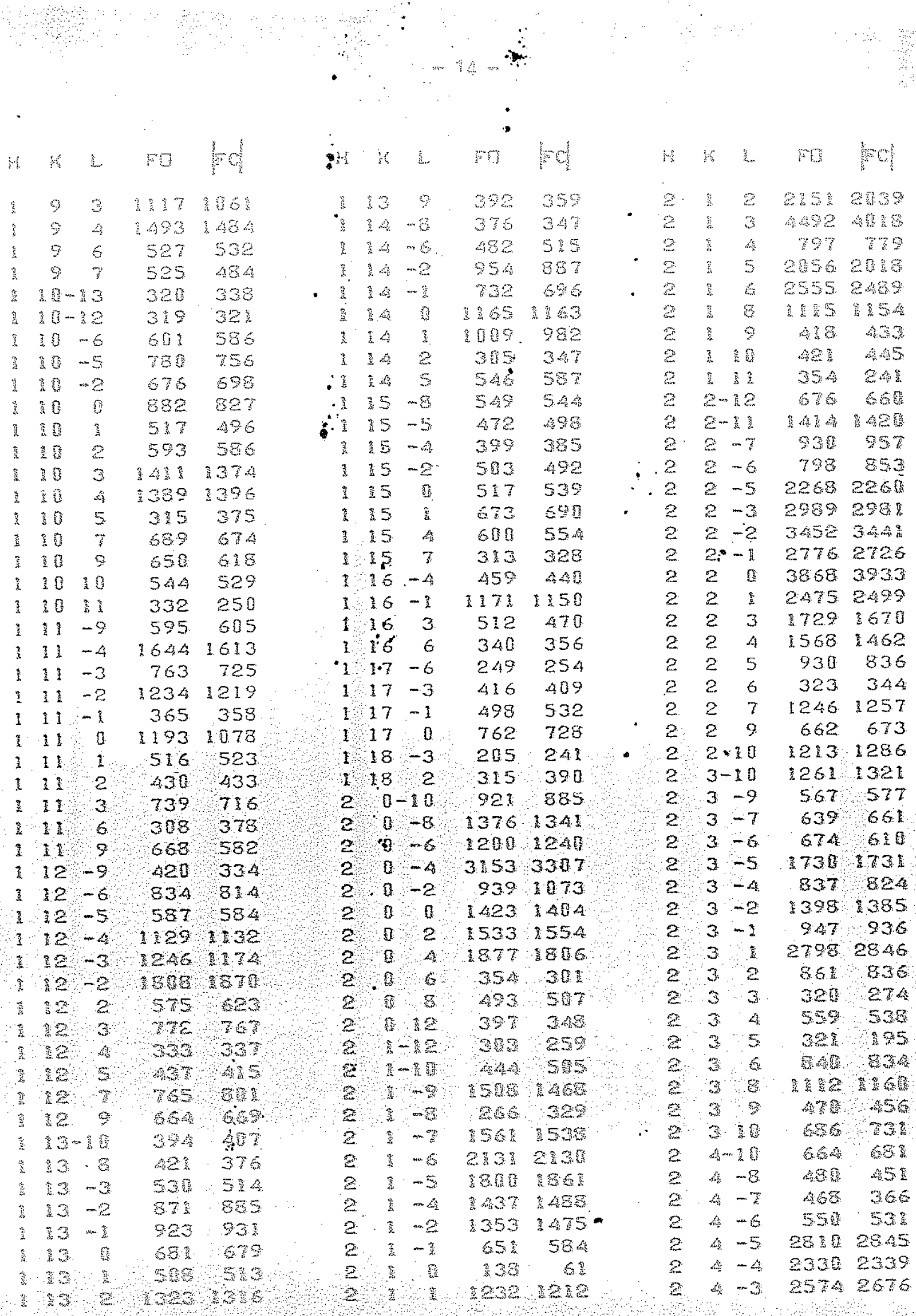




\begin{tabular}{|c|c|c|c|c|}
\hline 1 & $K$ & E. & $\mathrm{Fa}$ & 150 \\
\hline 2 & 4 & -2 & 2835 & 18 \\
\hline 3 & As & $-n$ & 295 & 295 \\
\hline 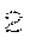 & 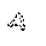 & 5 & 108 & 3.53 \\
\hline 8 & s & $\hat{\imath}$ & 387 & 343 \\
\hline & 4 & 8 & 325 & 328 \\
\hline 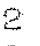 & 4 & 3 & 2287 & 128 \\
\hline & 4 & 4 & 673 & 679 \\
\hline & 4 & $G$ & 425 & 492 \\
\hline 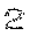 & 4 & 8 & 2356 & 2525 \\
\hline 2 & $\Delta$ & 20 & $2 \div 6$ & 37 \\
\hline 2 & 愴 & 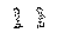 & 240 & 334 \\
\hline 2 & $5-$ & $: 2$ & 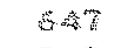 & 627 \\
\hline 2 & $5-$ & - 喜 & 519 & 544 \\
\hline 2 & & -5 & 2. 193 &  \\
\hline 2 & 5 & -9 & 叙堂 61 & 䇥慗 \\
\hline 2 & 5 & -8 & tan & 1666 \\
\hline 2 & 5 & -7 & 752 & 727 \\
\hline 2 & 5 & -5 & 2763 & 2755 \\
\hline 2 & 5 & -4 & 554 & 1613 \\
\hline 2 & 5 & -3 & 2179 & 2198 \\
\hline 2 & 5 & -2 & 3534 & 3510 \\
\hline 2 & 5 & -1 & 2742 & 2646 \\
\hline 2 & 5 & 0 & 1146 & +54 \\
\hline 2 & 5 & 1 & 5112 & $50 A$ \\
\hline 2 & 5 & 2 & 2025 & 2750 \\
\hline 2 & 5 & 3 & 2352 & 2265 \\
\hline 2 & 5 & $A$ & $33^{4}$ & 2256 \\
\hline 2 & 5 & 5 &  & 3.56 \\
\hline 2 & 5 & 6 & 588 & $6: 2$ \\
\hline 2 & 5 & 7 & 735 & $68 \pi$ \\
\hline 2 & 5 & 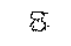 & 苔 520 & 2536 \\
\hline 2 & 5 & 9 & 373 & 424 \\
\hline 2 & 5 & 喜咅 & 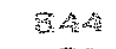 & 830 \\
\hline 2 & 6 & -3 & 40 & 568 \\
\hline 2 & 急 & -3 & 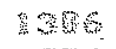 & 395 \\
\hline 2 & 6 & -3 & 528 & : 8 \\
\hline$z$ & 6 & -15 & $\mathrm{E}^{\mathrm{F}} \mathrm{F} S \mathrm{~S}$ & 278 \\
\hline 2 & 6 & $=-3$ & ba & $2 z^{2}$ \\
\hline a & 6 & -5 & 848 & so \\
\hline 2 & * & $\rightarrow$ & $\sin$ & 52 \\
\hline 2 & $\varepsilon$ & P & 2.5 & (1595 \\
\hline e & 5 & $\sqrt{\mathrm{Z}}$ & 764 & 853 \\
\hline 2 & 6 & 2 & 339 & 832 \\
\hline 2 & 6 & 3 & 427 & 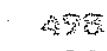 \\
\hline 2 & 6 & 4 & 35 & 328 \\
\hline 2 & 6 & 5 & 3 等 & 238 \\
\hline 2 & 6 & 6 & 恋 & tas \\
\hline 2 & 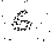 & 3 & $\mathrm{~s}^{2}$ & me \\
\hline 2 & s & 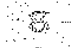 & at & tw \\
\hline . & s & is & 27 & rat \\
\hline
\end{tabular}

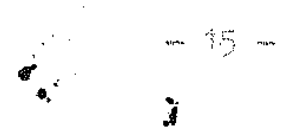

\begin{tabular}{|c|c|c|c|c|c|c|c|c|c|c|}
\hline 3 & औ & 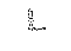 & $\mathrm{BO}$ & aco & & $\xi$ & $\mathcal{K}$ & L & W & $F^{2}$ \\
\hline 2 & 6 & 12 & 336 & 367 & & 2 & In & 8 & 58 & 538 \\
\hline 2 & 7 & -6 & 4.22 & 1436 & & 2 & 影 & $\approx$ & $\mathrm{W}$ & 98 \\
\hline $\mathrm{g}$ & 7 & -4 & 209 & $2: 3$ & &  & 期 & 4 & 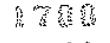 & 言管落食 \\
\hline & 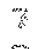 & w然 & $\sqrt{2} 48$ & s A A & & 2 & 80 & $\hat{3}$ & 68 & 金的筧 \\
\hline 8 & 7 & $m A$ & 86 & 70 & & 2 & 20 &  & $53 \pi$ & 5 \\
\hline 2 & 7 & -3 & 3676 & 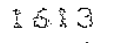 & & 2 & $\sqrt[3]{3}$ & 9 & 422 & $46 A$ \\
\hline 2 & 7 & -2 & 285 & 350 & & 2 & 42 & 21 & $7 \mathrm{n}^{2}$ & 25 \\
\hline 2 & 7 & -3 & 544 & 523 & & 2 & $8:$ & 管 & 55 & $a^{2}$ \\
\hline 2 & $\frac{7}{3}$ & 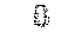 & 922 & 806 & & 2 & 食昆 & -4 & 233 & 376 \\
\hline 2 & 7 & 点 & 量 60 & 1 69 & & 2 & 15 & -3 & 49 & $=28$ \\
\hline 2 & 7 & 2 & 1895 & 99 & & 2 &  & -2 & 52 & 被 \\
\hline 2 & 7 & 3 & 220 & $2: 75$ & & 2 & 霍䈍 & 箸 & 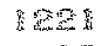 & 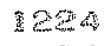 \\
\hline 2 & 7 & $5^{\circ}$ & 573 & 6籍竞 & & 2 & $\hat{1} \bar{i}$ & 2 & s 49 & 40 \\
\hline 2 & $\frac{7}{v}$ & 6 & 545 & $\cong 5 \%$ & . & 2 & 告 $\sqrt{3}$ & 3 & 36 & 870 \\
\hline 2 & $\bar{\xi}$ & 7 & 406 & 472 & & 2 & 篦䍗 & 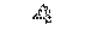 & 83 & 5 \\
\hline 2 & 7 & 8 & 533 & 573 & & 2 & 萦 & 5 & 736 & 761 \\
\hline 2 & 8 & -8 & 9程0 & 866 & & 2 & I & 6 & 537 & 606 \\
\hline 2 & 8 & -7 & §569 & 2629 & & 2 & 焉 & 7 & $=290$ & 985 \\
\hline 2 & 8 & -5 & 2139 & 2069 & & 2 & $\mathbb{E}$ & 8 & 557 & 536 \\
\hline 2 & 8 & -4 & 374 & 273 & & 2 & $3 k$ & $\dot{9}$ & 490 & 482 \\
\hline 2 & 8 & -3 & 973 & .8004 & & 2 & $2-$ & 2 & 405 & 352 \\
\hline 2 & 8 & -2 & 327 & 266 & & 2 & 12 & -8 & 802 & 35 \\
\hline 2 & 8 & 0 & 1783 & 1776 & & 2 & 12 & -3 & 523 & 518 \\
\hline 2 & 8: & $=1$ & 1051 & 959 & & 2 & 2 & -2 & 1500 & 1529 \\
\hline 2 & $\xi$ & 2 & 2235 & $225, \pi$ & & 2 & 2 & -2 & 362 & 45 \\
\hline 2 & 3 & 3 & I0 & 5017 & & 2 & 12 & 9 & 943 & 9646 \\
\hline 2 & 8 & $\Delta$ & 2089 & 2086 & & 2 & $\tilde{a}$ & 3 & 395 & 369 \\
\hline 2 & 6 & 5 & 3756 & 799 & & 2 & 蒿2 & 6 & 326 & 鈗正 \\
\hline 2 & 8 & 5 & 2778 & 2923 & & 2 & 12 & 4 & 655 & 64 \\
\hline 2 & s & 9 & 52 & 348 & & 2 & 2 & es & 786 & 764 \\
\hline 2 & 9 & $-g$ & 632 & $58 \pi$ & & 2 & 2 & 5 & 526 & 532 \\
\hline 2 & 9 & $\dot{m}$ & 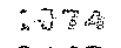 & 3: 5 & & 2 & 2 & 意唯 & 432 & 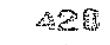 \\
\hline 2 & 9 & $-b$ & 2467 & 2An & & 2 & 3 & -9 & 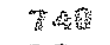 & 25 \\
\hline 2 & 9 & -5 & 58 & 73 & & 2 & 3 & $-A_{t}$ & 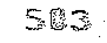 & 5 愈㤎 \\
\hline 2 & $\$$ & 2 & 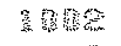 & b? & & 2 & 39 & -2 & 359 & 3. \\
\hline 2 & \% & -0 & 38 & 35 & a & 2 & 32 & 致 & 46 & 52 \\
\hline 2 & 9 & 沗 & 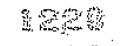 & 24 & - & 2 & 3 & 3 & 6 연 & 65 \\
\hline 2 & 9 & 藻 & 5 & A & • & 2 & 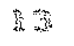 & $A_{1}$ & 352 & 37 \\
\hline 2 & 9 & 2 & 85 & 9 & & 2 & 霍䋊 & 9 & 659 & 50 \\
\hline 2 & 3 & 磨 &  &  & • & 2 & 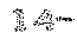 & 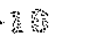 & sand & 36 \\
\hline 2 & 9 & 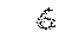 & 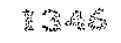 & 等3 3 & & 2 & 零 & -5 & 398 & $28 \mathrm{E}$ \\
\hline $2^{\circ}$ & 9 & 䇣 & 54 & 58 & & 2 & 绿 & -6 & A & $n 2=$ \\
\hline 2 & 9 & e &  & $6 \%$ & & $\approx$ & A & -2 & tis 6 & 828 \\
\hline 2 & 9 & 雭触 & 32 & 296 & & 2 & 答 & ra & bso & 2. \\
\hline 2 & 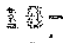 & 喜嵒 & 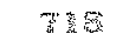 & 69 & & 2 & 䈉 &  & 978 &  \\
\hline 2 & 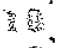 & $-\xi$ & 53 & $5 \mathrm{~b}$ & & 2 & $15^{\circ}$ & $-g$ & 286 & 206 \\
\hline 2 & 等 & $\frac{-3}{5}$ & 政 & 95 & & 2 & 5 & -4 & A &  \\
\hline 2 & I & 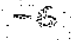 & 132an & 等55 & & 2 & 5 & $\therefore$ & 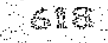 & $\cos ^{2} 5$ \\
\hline 2 & a & -5 & bx & 6s & & 2 & 5 & 2 & $x^{3}$ & Hen \\
\hline 2 & (1) & -1 & tos & to & & 2 & 3 & a & 34 & 53 \\
\hline
\end{tabular}


$16 \cdots$

\begin{tabular}{|c|c|c|c|c|c|c|c|c|c|c|c|c|c|}
\hline a & 2 & $\mathrm{mo}$ & $\mathrm{Pec}$ & 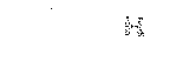 & $\mathbb{F}^{\prime}$ & 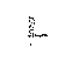 & 50 & Pro & 4 & M & E. & $\mathrm{F}$ & Fer \\
\hline & 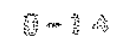 & 53 & 446 & 3 & 3 & -5 & 53 & 6 & 3 & 5 & 6 & 232 & 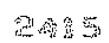 \\
\hline & 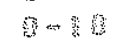 & 322 & 355 & 3 & 3 & -4 & 392 & 1528 & 3 & 5 & \% & 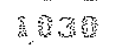 & Q \\
\hline & $2-6$ & 822 & $7 \hat{3}$ & 3 & 3 & -3 & 329 & 325 & 3 & 5 & 20 & $4 E$ & $4 \mathbb{E g}$ \\
\hline & $0-4$ & 505 & 1754 & +3 & 3 & -2 & $\$ A B A$ & 1376 & 3 & 6 & -5 & 629 & 50 \\
\hline & $6-2$ & 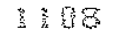 & \$98 & 3 & 3 & $-\pi$ & 803 & $\mathrm{TH}$ & 3 & 6 & -8 & 495 & \\
\hline & 核 & 350 & 1975 & 3 & 3 & $\mathrm{~B}$ & $39^{\circ}$ & 383 & 7 & 6 & -5 & 568 & 28 \\
\hline & 2 & 1327 & 2809 & 3 & 3 & $\hat{\varepsilon}$ & 2769 & $2 \sec$ & 3 & $\Leftrightarrow$ & $-\vec{s}$ & 235 & inis \\
\hline & $\hat{A}$ & 938 & 933 & 3 & 3 & 2 & 290 & 369 & 3 & 6 & $-1^{*}$ & 925 & \\
\hline & 6 & 278 & 266 & 3 & 3 & 3 & 315 & 268 & 3 & 6 & 0 & 338 & \\
\hline & 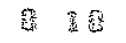 & 562 & 5,6 & 3 & 3 & a & ATP & 430 & 3 & 6 & $\hat{\mathfrak{s}}$ & 232 & \\
\hline & 1e & 725 & t5e & 3 & 3 & $s$ & 5. & 516 & 3 & 6 & 2 & 255 & \\
\hline & $1 \sim \frac{9}{2}$ & 50 & 522 & 3 & 3 & 7 & 527 & 499 & 3 & 6 & 3 & 453 & \\
\hline & 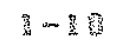 & 917 & 990 & 3 & 3 & $\mathrm{~g}$ & $A 5 d$ & $A \Delta 9$ & 3 & 宛 & 4 & mes & I \\
\hline & ? -9 & 32 & 27 & 3 & 3 & 9 & 6.42 & 643 & 3 & 6 & 6 & 1798 & beat \\
\hline & $\mathbb{1}-7$ & 428 & 359 & 3 & $4-3$ & 12 & 669 & 712 & 3 & $\dot{6}$ & 7 & 的的7 & ges \\
\hline & $5-6$ & ress & 729 & 3 & $\Delta-\mathbb{Z}$ & $\frac{1}{3}$ & $A 17$ & $\sqrt{47}$ & 3 & 5 & $\hat{\mathbb{1}}$ & 652 & 6 \\
\hline & -5 & 722 & 636 & 3 & $m-1$ & 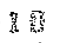 & 583 & 636 & 3 & $T_{\bar{y}}$ & - & 293 & 3AS \\
\hline & $1 \quad-4$ & 1825 & 1549 & 3 & $\Delta_{3}$ & -9 & 242 & 2236 & 3 & 7 & -8 & 颉了 & 8537 \\
\hline & $\begin{array}{ll}5 & -3\end{array}$ & 453 & 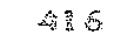 & $3:$ & 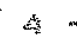 & -8 & 585 & 58 & 3 & 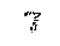 & -7 & 3310 & 3353 \\
\hline & $1-2$ & 209 & 239 & 3 & $\therefore$ & -6 & 笋面 8 & 752 & 3 & T & -6 & 377 & 1758 \\
\hline & $\hat{s}-\hat{s}$ & 108 & 2035 & 3 & $A$ & -5 & 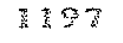 & 1296 & 3 & 7 & -3 & 255 & 273 \\
\hline & $\hat{\imath}$ & $\mathbb{1}: \mathbb{R}$ & 2090 & 3 & A & $-\Delta \hat{i}$ & 575 & 582 & 3 & $T$ & $-\hat{i}$ & 55.4 & 529 \\
\hline & $\frac{\bar{t}}{2}$ & 277 & 295 & .3 & 邻 & -3 & 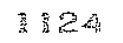 & \$D: & 3 & 7 & $\bar{s}$ & SAB & 572 \\
\hline & 3 & 1980 & $20+4$ & $\cdot 3$ & A & -2 & 1394 & 8296 & 3 & $\pi$ & 4 & 1766 & 8819 \\
\hline & 3 & 2122 & 2039 & $: 3$ & A & $-\bar{\Sigma}$ & 807 & 760 & 3 & 7 & 5 & 5502 & 3492 \\
\hline & $A_{A}^{A}$ & 34 & $32 A$ & 3 & 4 & $\sqrt{0}$ & 933 & $9 n$ & 3 & 3 & 6 &  & 423 \\
\hline & n & 585 & 245 & 3 & 4 & 2 & 392 & 48 & 3 & $\mathrm{~g}-$ & 一昰彩 & 729 & 69 \\
\hline & 3 & 806 & 854 & 3 & 2 & 2 & $55 ?$ & $58 ?$ & 3 & 6 & $-\xi$ & 政 & 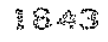 \\
\hline & 12 & 306 & 259 & $\therefore$ & 今 & 4 & 889 & 915 & 3 & B & $-A_{0}$ & 666 & 564 \\
\hline & $2-8$ & 345 & 326 & 3 & 索 & 3 & 325 & 3.99 & 3 & 会 & -2 & 563 & 556 \\
\hline & $2-5$ & 塕籍7 & 9.3 & 3 & $A$ & 7 & 85 & 782 & 3 & 8 & 筙 & $58 \pi$ & 53.4 \\
\hline & $2-5$ & 453 & 532 & 3 & 4 & 9 & 639 & 654 & 3 & 8 & $2^{\circ}$ & 652 & 629 \\
\hline & $2-\frac{\Delta}{4}$ & 3893 & 2933 & 3 & 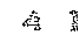 & 筷鼠 & 380 & 435 & 3 & $\mathrm{E}$ & 3 & 45 & 391 \\
\hline & $2^{-3}$ & 1655 & $357 \%$ & 3 & $5-8$ & 12 & sale & 482 & 3 & $\mathbb{s}$ & $t_{4}$ & 2685 & 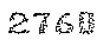 \\
\hline & $2-2$ & 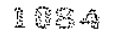 & 255 & 3 & $5-3$ & 3 & 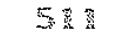 & 532 & 3 & $\mathrm{~B}$ & 5 & 5.536 & 259 \\
\hline & $2-3$ & 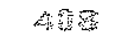 & 450 & 3 & 5 & -9 & 1229 & Re霖 & & 8 & 5 & 55 & 802 \\
\hline & 2 & 723 & 7) & 3 & 5 & -8 & 580 & 626 & 3 & 9 & $\sqrt{15}$ & 235 & 28 \\
\hline & 2 & 3337 &  & 3 & 5 & -7 & 35 &  & 3 & & 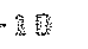 & 575 & 67 \\
\hline & 2 & 290 & 25 & $\Rightarrow$ & 5 & -6 & reds & 至29 & 3 & 9 & -5 & $32 x^{2}$ &  \\
\hline & 3 & 52 & $55 \%$ & 3 & 5 & -5 & 65 & 723 & 3 & 9 & $-\frac{7}{5}$ &  &  \\
\hline & 角: & Tas & 叐紫零 & 3 & 5 & $-\mathrm{s}$ & 26 & ens & 3 & 9 & -6 & nestat & 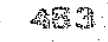 \\
\hline & औ & 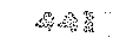 & קहs & 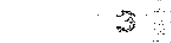 & 5 & $-\frac{5}{3}$ & 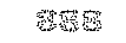 & T: 53 & 3 & 9 & -3 & 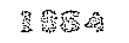 & 93 \\
\hline & 2 & 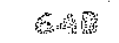 & 65 & 3 & $5-$ & -2 & $28 n$ & 26 & 3 & 9 & $-m$ & 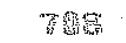 & 75 \\
\hline & 28 & 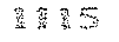 & 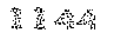 & 3 & 5 & $-\frac{1}{8}$ & 227 & 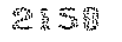 & 3 & 9 & $\frac{1}{5}$ & 75 & $76 \sin ^{2}$ \\
\hline & 9 & 578 & 5.74 & 3 & 5 & 到 & 925 & $9 \sqrt{2}$ & 3 & 9 & 2 & 筀6 & 25 \\
\hline & $3-5$ & 392 & 383 & 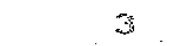 & 2 & \# & An & 能GS & & $y$ & 3 & 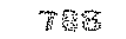 & 78 \\
\hline & $3=\frac{1}{3} \sqrt{1}$ & 369 & 779 & 3 & 5 & $\approx$ & sese & n:B: & 3 & 9 & 4 & 管 40 & 5535 \\
\hline & $3-9$ & 432 & 469 & 3 & 5 & 3 & 435 & 45 & & 9 & 5 & 272 & 358 \\
\hline & $3-7$ & $73 \mathrm{~A}$ & 689 & 3 & 5 & 4 & 188 & 953 & 3 & 9 & 6 & 9 de & 29 \\
\hline & $3-6$ & 35,4 & 36 & 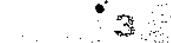 & 5 & 5 & 7 & 520 & 3 & 9 & 7 & 624 & Bss \\
\hline
\end{tabular}




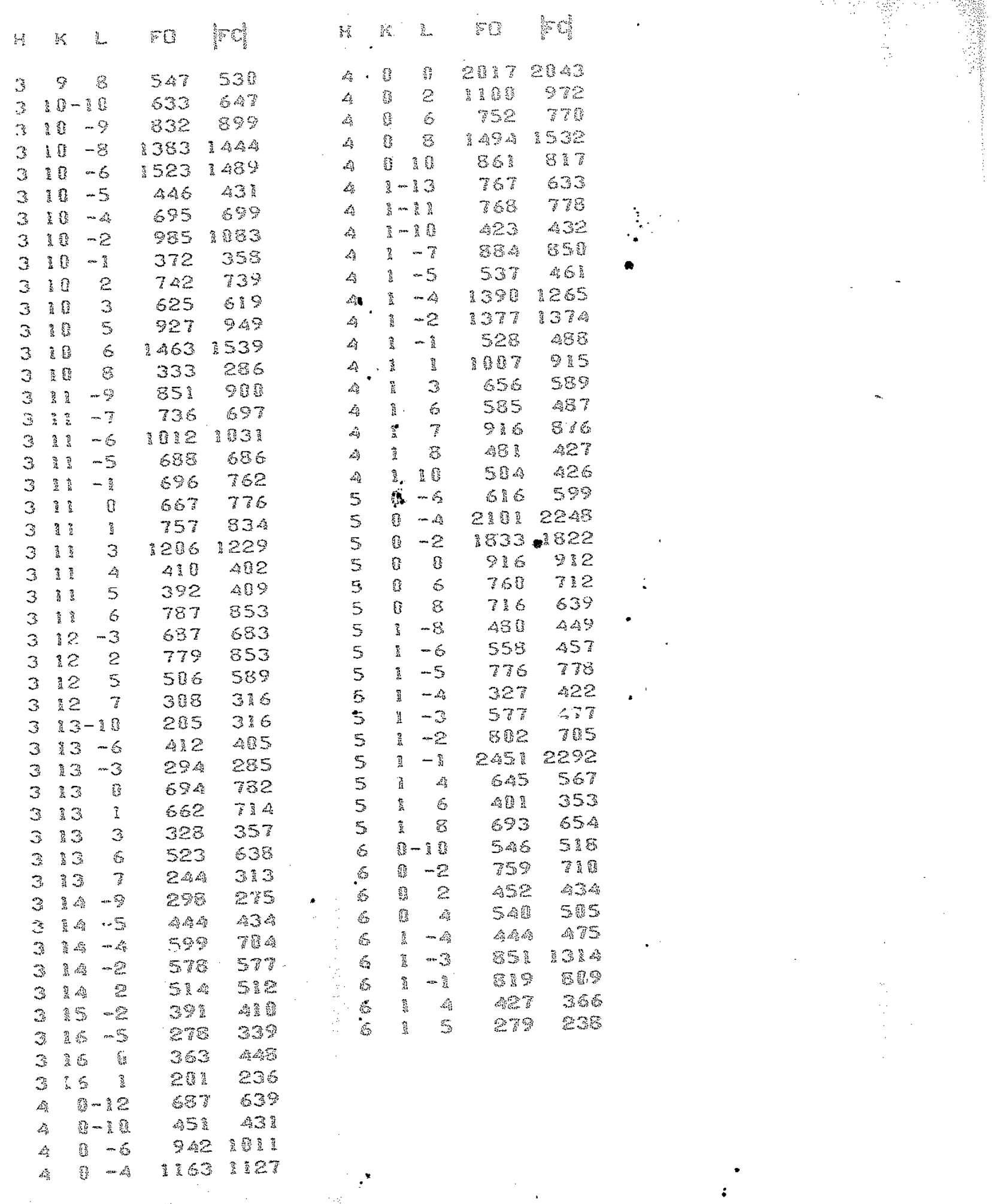

